\title{
Génesis y tensiones de la Comunidad Societal de Parsons
}

\author{
Francisco J. Cantamutto*
}

\begin{abstract}
Resumen
El presente artículo explica la génesis y desarrollo del concepto de Comunidad Societal del sociólogo funcionalista Talcott Parsons. Aunque según Alexander (1989), para Parsons cada cambio en su esquema de análisis es un desprendimiento lógico de su estructura básica, la Comunidad Societal implicó cierta novedad respecto de sus planteos iniciales. El rastreo del concepto se hace, entonces, siguiendo la obra del propio Parsons, buscando explicar los cambios teóricos por donde se arriba a este concepto. Se presentan al final dos tensiones del propio concepto, respecto de la inclusión de grupos no miembros y el uso de la coerción.
\end{abstract}

Palabras clave: Comunidad Societal - orden social - Parsons.

\begin{abstract}
This article explains the genesis and development of the concept of Societal Community, created by functionalist sociologist Talcott Parsons. Although according to Alexander (1989) for Parsons each change in his scheme of analysis is a logical evolution of its basic structure, the Societal Community involves certain novelty. The tracking of the concept is thus done following the works of Parsons, seeking to explain the theoretical changes through which this concept arose. Finally, the article presents two internal tensions of the concept itself, regarding the inclusion of non-member groups and the use of coercion.
\end{abstract}

Keywords: Societal Community - social Order - Parsons.

* Licenciado en Economía, Universidad Nacional del Sur, Argentina. Máster en Ciencias Sociales y Doctor en Investigación en Ciencias Sociales con mención en Sociología, FLACSO, México. 


\section{INTRODUCCIÓN}

Los sociólogos clásicos de la segunda generación escriben entre fines del siglo XIX y principios del XX, cuando los cambios propulsados por las revoluciones industrial y democrática habían comenzado a afianzarse (Alexander, 1990). La "idea elemento" (Nisbet, 1996) de Comunidad sirvió para la formulación de un par dicotómico con Sociedad, fructífero en términos de desarrollo de la disciplina sociológica. Tönnies ofrece la primera proposición en estos términos y será retomado a partir de allí en diferentes autores y obras (Sasín, 2012: 73).

De Marinis (2010b) propone la posibilidad de aprehender esta dicotomía en tres claves complementarias de lectura. En primer lugar, esta propuesta está asentada en la intención de dar cuenta del proceso de cambios detectados por los autores clásicos como signo de su época (tipo histórico). La instalación del doble par permitía describir un cambio en una dirección específica (quizás aún imbuidos de las nociones lineales de tiempo tan típicas por entonces), que iba desde la Comunidad a la Sociedad. En segundo lugar, la identificación de características prototípicas de las relaciones entre los hombres y mujeres, para la descripción "aséptica" de la realidad en función de la presencia o no de ciertos parámetros bien definidos (tipo ideal). Finalmente, incluso cuando hubieran pretensiones de neutralidad valorativa, la dicotomía hacía las veces de fuente de crítica a la realidad presente, definiendo aquello que ya no está o aquello que falta, y por ello mismo, sirviendo de base para la posibilidad de buscar ciertos cambios (tipo utópico).

El mismo autor propone visualizar la (re)aparición del concepto en la etapa ligada a los años del Estado de Bienestar o welfarismo (De Marinis, 2005). El teórico por excelencia de la sociología de esta etapa es, para bien o mal, Parsons. La tematización de la comunidad en Parsons llega relativamente "tarde": es en las obras de sus últimas etapas que aparece explícitamente el concepto de Comunidad Societal (en adelante, CS). De hecho, será en el libro La Sociedad: perspectivas evolutivas y comparadas (1966) donde por primera vez se enuncie este concepto. Y será propuesto en un sentido muy diferente de los autores clásicos.

Según el propio Parsons afirma (1986: 55), la dicotomía Comunidad-Sociedad no permite una buena lectura de la realidad, principalmente por las reminiscencias idealizantes de un pasado que cambió. Sin la nostalgia por las relaciones "cálidas" adjudicadas a la Comunidad, ni el pesimismo por el desgajamiento de la Sociedad, Parsons celebró el (supuesto) pluralismo de su tiempo, en un optimismo endémico (Holton y Turner, 1986). Pero, lo que es más, con su propuesta conceptual de la CS Parsons logra sintetizar -paradójicamente- ambos polos de la dicotomía (Sasín, 2012; Sciortino, 2005). Parsons se encontraba fascinado con la capacidad de la sociedad contemporánea para lidiar con la tensión emergente de la desigualdad producida por el mercado y la igualdad propuesta por la democracia (Boasso, 1980; Holton y Turner, 1986), en un optimismo que rayaba lo celebratorio o apologético (Alexander, 2005). De Marinis (2010b) señala que, de las tres claves de comprensión arriba señaladas, con Parsons la que aplica con más justeza es la de tipo utópico: ¡como conciencia de la racionalidad keynesiana! 
Sin embargo, no sería cierto afirmar que el concepto irrumpe desde el vacío en la obra del autor. Como muestran Fox et al. (2005), se trata de una lenta maduración dentro de las líneas fundamentales de su pensamiento. Sciortino (2007), por su parte, señala su entrelazamiento con otras reflexiones de Parsons en esa época -medios generalizados de intercambio, teoría de la evolución-. De Marinis (2010a) señala que existen al menos dos líneas de continuidad en la obra de Parsons, ligadas a sendos problemas que lo aquejaron permanentemente: el problema del orden (y su solución hobbesiana) y el de los componentes no racionales de la acción.

El presente artículo explica la génesis y desarrollo del concepto de CS dentro de la obra de Parsons, partiendo de los planteos iniciales del autor hasta llegar a su esquema evolucionista. Se plantean al final dos tensiones internas al concepto, que Parsons no resuelve y que, según entendemos, ponen cota a los alcances democráticos que el autor le atribuye a su propuesta.

\section{EL PROBLEMA DEL ORDEN Y LA SOLUCIÓN UTILITARISTA}

En sus primeras obras, Parsons (1968) discutió abiertamente con las tesis utilitaristas para explicar la sociedad. Los planteos utilitarios retoman el sensualismo de la Antigüedad, que asume que todo acontecer existencial es evaluado en términos de placer o displacer provocado al individuo. En su versión moderna, esta corriente propone como guía de la acción a la maximización de la utilidad individual, proponiendo al individuo como mejor juez para tal determinación. Según Parsons (1967a, 1968), cuatro serían las características de este enfoque: i) Atomismo: asume como unidad de análisis al individuo, estando este desposeído de capacidad de imponerse al resto de modo unívoco; ii) Racionalismo: los individuos tienen plena racionalidad en la determinación de los elementos que componen la situación donde se maximiza el alcance de fines; iii) Carácter fortuito de los fines: no hay dentro de la teoría ninguna restricción que permita predecir los fines que los individuos perseguirían, son determinados exógenamente y, por tanto, aleatorios para el sistema; y iv) Empirismo: toma por método la acumulación de casos o experiencias, proyectando a la totalidad social características halladas (y por ello, solo válidas) en las partes.

Debido a que el único criterio para aceptar una regla general es -para el utilitarismo- que esta aporte en todos los casos a la maximización de la utilidad personal, lo que es altamente improbable, no podríamos prever la aceptación de ninguna regla de aplicación generalizada, universal (Münch, 1982). El enfoque utilitarista sería incapaz de explicar el orden social, es decir, la existencia de cierta estabilidad empírica en la organización (cualquiera esta sea) del mundo social. Y sin embargo, la sociedad no se desintegra: ¿cómo se explica esto?¹.

Esta situación hobbesiana solo sería solucionada a partir de la imposición de un orden por vía coercitiva (Alexander, 1989). Sería, sin embargo, una falsa solución, que traslada

1 Sciortino (2007) señala que esta preocupación de la integración social es el centro de la reflexión parsoniana, algo que la teoría social posterior habría descuidado. Esta idea es Ilamativa, porque, por ejemplo, los esfuerzos de Habermas (1998) y de Luhmann (1998) están claramente centrados en esta explicación por medio de la comunicación. 
el problema a la disputa por el control del órgano con capacidad de imposición coercitiva (Münch, 1982). Por lo demás, no altera sustancialmente el problema: la aplicación de sanciones solo agrega un elemento más en la ecuación de costos y beneficios del cálculo individual. La estabilidad no se alcanzaría por esa vía, lo que constituye el dilema (aporía) utilitarista: la necesidad de oscilar entre una salida liberal (postular pequeñas unidades interactuantes con capacidad mutua de control) y una salida absolutista (que una unidad tenga capacidad unívoca de imponerse al resto).

Una salida a este dilema sería la construcción de un "nosotros" (Münch, 1983): un marco de referencia colectivo, formado a partir de la interacción discursiva y la construcción de consenso. Este fue el camino de la primera teoría (voluntarista) de la acción de Parsons (1968): constituir una tradición normativa común compone un límite a la acción al constreñir el campo de elegibles. Parsons intentó así combinar el racionalismo utilitarista con la importancia de la normatividad en la acción (tesis de la convergencia). Para esto rastreó en Weber, Durkheim, Marshall y Pareto los elementos que le permitían explicar la emergencia de la teoría voluntarista. Según esta, las normas no resultan una exterioridad al individuo actuante, sino que forman parte constitutiva de la definición misma de la situación y los fines que el actor persigue. La norma, internalizada mediante la socialización, limitaría, a la vez que permite, los cursos de acción. De este modo, el conjunto de los fines asequibles y los medios utilizables se restringen, desde el propio actor. A partir de la existencia de patrones de valor compartidos, los individuos incorporan ciertos cursos de acción como motivaciones particulares, lo que permite establecer el orden como una premisa explicada (en lugar de una conclusión derivada). El orden social estaría entonces basado idealmente en la integración en un sistema de valores comunes (Parsons, 2007: 69; Sasín, 2012: 81).

La distancia respecto de un normativismo "a secas" estaría en el elemento voluntario del actor. Así como la propuesta kantiana entiende que las reglas morales requieren para su aplicación no solo de razón (distinguir entre el Bien y el Mal) sino también voluntad (de seguir el camino que la razón distingue); Parsons entendía que la norma propone valores que motivan, pero que el actor siga o no este camino es un problema volitivo, no una imposición externa. Los sujetos, señaló Parsons (1968: 118), no responden solo a estímulos, sino que tratan de ajustar su acción a modelos deseados. Esta normatividad afecta de conjunto a los elementos del acto-unidad: a la definición de la situación (condiciones y medios) tanto como a los fines perseguidos. La existencia misma de elementos normativos explica el orden en la sociedad. La pregunta planteada desde el principio es, entonces, de dónde emergen los "modelos deseados". Evidentemente, su origen no puede ser el individuo: es ineludible una referencia a los demás (los otros). Esto llevó a Parsons a problematizar y generalizar su esquema original.

\section{DEL ACTO UNIDAD AL PROBLEMA DE LA INTEGRACIÓN}

Luego de la Estructura de la acción social (1937), Parsons publicó varios trabajos, muchos de estos ensayos en referencia a problemas políticos concretos de su época. En ellos se perfila con claridad un razonamiento ampliado, luego, el rol de la socialización para la integración 
entre normas y pautas de comportamiento. En varios de los artículos compilados como Ensayos de teoría sociológica (1967a [1947]) propone a la educación y la propaganda como las principales formas de afectar los sentimientos y las pautas de referencia de los sujetos. En su siguiente gran obra, El sistema social (1951), se produce un cambio de atención: el proceso de interacción constituye un esquema relacional permanente, constituyendo un sistema. Fue un paso importante en el giro -nunca completo- desde una teoría de la acción a la reflexión respecto de la teoría de sistemas (Luhmann, 1995; Parsons, 1977: 234; cf. Almaraz, 1979).

La unidad básica del acto-unidad era analizada ahora bajo la organización de un sistema: los actores interactúan sobre la base de ciertas estructuras de relación, según se encuentran ocupando posiciones (estatus) que suponen ciertos procesos (rol), como atributos del propio sistema (Parsons, 1966: 33-34). La orientación motivacional de los actores (búsqueda de gratificación o de evitar la privación) depende de ciertas pautas, que, por su relativa estabilidad, no parecen depender de la situación. La estabilidad de ciertos símbolos y valores remite a una característica del sistema y no de la acción contingente del actor. De hecho, el actor, imbuido en interacciones cotidianas con otros actores, comienza a esperar que esas pautas se validen en la acción de los otros, logrando de esa forma adecuar sus expectativas.

Parsons (1966: 16) propuso aquí las cuatro dimensiones del sistema general de la interacción humana: la personal, la social, la cultural y la biológica ${ }^{2}$. La acción humana, marco de referencia común, se compone a partir de elementos de los cuatro subsistemas. El orden se produce por la integración de las motivaciones de los actores con criterios normativos culturales, la estabilidad de la interacción es producto de criterios de valor comunes (1966: $25,49)$. La integración en el sistema total de la acción es un compromiso entre las tensiones de consistencia de sus componentes sociales, culturales y de personalidad, compromiso nunca perfecto $(1966: 26-27)^{3}$.

En otros términos, el sistema social requiere que los actores incorporen pautas valorativas comunes a sus personalidades, que guíen la acción en ciertos sentidos y no en otros. Para motivarlos correctamente, el sistema recurre a la socialización: lograr que el actor incorpore a la personalidad pautas normativas necesarias para la subsistencia del sistema. Es indistinto si los actores incorporan las normas por conveniencia o por introyección. El sistema deberá encontrar gratificaciones y compensaciones para las expectativas generadas en los actores, cuya realidad no necesariamente coincidirá con el ideal proyectado. Necesita, además, cubrir las necesidades básicas de los actores. En este sentido, debe resolver la asignación de recursos del modo de maximizar las capacidades actuales y futuras. La integración y la

2 Esta última ya aparecía aunque menos desarrollada en el planteo de la Estructura de la acción social (1968: 85).

3 Sciortino (2007) señala que esta tensión irresuelta es sustancial al funcionamiento de la sociedad, y no una categoría residual, con lo que la acusación a Parsons respecto de su fascinación por el orden social, desatendiendo los cambios, pareciera algo exagerada. No obstante, un lector atento de la obra de Parsons ha señalado que, a pesar de reconocer las tensiones, aparecen como contingentes ante la necesidad funcional de estabilidad (Luhmann, 1995: 44). 
asignación de recursos serán los principales problemas del sistema social como tal (Fox, Lidz y Bershady, 2005).

Según se enuncia en El sistema social (1966: 36-40), los prerrequisitos funcionales de los sistemas sociales son: i) cumplir las condiciones de funcionamiento orgánico; ii) generar las motivaciones de rol y evitar la conducta lesiva; y iii) lograr el mantenimiento de pautas culturales que garanticen un mínimo de orden. Están aquí enunciadas funciones que entablan relaciones directas entre los distintos subsistemas. Según realzaremos más tarde, Parsons hace explícita la doble dimensión involucrada: la socialización (para internalizar las pautas) y el control social (para quien no cumple lo anterior). Es decir, la propuesta de un orden no basado en la coerción para solucionar el problema utilitario choca contra las necesidades funcionales del sistema.

Más allá de esta ambivalencia, la propuesta de Parsons es clara. El sistema social requiere para su sostenimiento de lograr cierta integración, cierto compromiso entre los valores del actor y los del sistema. Los prerrequisitos funcionales adoptarán en las siguientes obras de Parsons una autonomía propia, al asociarse a las funciones de los diferentes subsistemas (Mitchell, 1967: 55-58). Aun cuando no encontramos aquí la noción de comunidad de manera explícita, comenzamos a verificar que las necesidades de estabilidad giran de énfasis hacia la estructura de la interacción, que -por definición- involucra a más de un actor. Es decir, el problema del orden no tiene solución en la escala del actor: es necesaria una perspectiva sistémica.

\section{EL ANÁLISIS TETRADIMENSIONAL}

Parsons desarrolló una generalización empírico-conceptual en el modelo conocido por el acrónimo AGIL. Münch $(1982 ; 1990)$ propone entender este esquema como la continuidad de los elementos del acto-unidad, combinando nuevos ejes como se ve en el diagrama. Las iniciales refieren a los cuatro problemas o funciones del sistema general de la acción, caracterizado como un sistema abierto (Almaraz, 1979; De Marinis, 2010b; Fox, Lidz y Bershady, 2005; Luhman, 1995).

A. Adaptación: refiere al control generalizado sobre los intercambios con el ambiente, orientado a la satisfacción de las exigencias situacionales. El sistema tiene que adaptarse a su entorno y lograr que este se adapte a él. Requiere la generación de recursos y su asignación entre los actores. El organismo conductual se especializa en el cumplimiento de esta función. En relación con la acción, se encuentra en el máximo de complejidad simbólica (la necesidad de interpretar es mayor) y mayor contingencia, es decir, menor control por parte del sistema: se trata de ajustar los medios (disponibles) para alcanzar ciertos fines (parcialmente generados por el ambiente), ambos en un marco de amplia variabilidad.

G. Logro de metas: son los procesos de organización de las actividades en la forma de esfuerzos concertados por alcanzar ciertas metas primordiales. Definir esas metas y alcanzarlas es función del sistema de la personalidad. Respecto de la acción, implica menor contingencia (mayor control) y mantiene una elevada complejidad simbólica: se 
trata del ajuste más regulado de los objetivos a alcanzar, pretendidamente controlados por la socialización de las personas (que, en cualquier caso, tienen que interpretar la situación en la que se encuentran).

I. Integración: refiere a los procesos de ajuste mutuo entre unidades sociales, su interrelación, para garantizar la lealtad, la adhesión y la interdependencia. Implica el establecimiento de prioridades entre unidades, y la institucionalización de un orden normativo común junto con mecanismos de control social. La integración es la contrapartida de la adaptación, en el sentido de organizar el ambiente interno del sistema. La diferenciación de unidades en el sistema requiere que se desarrollen mecanismos que creen solidaridad y evite los conflictos. Es la tarea que cumple el sistema social. La acción aquí tiene menor contingencia y menor complejidad simbólica: las normas están allí para ser cumplidas, y existe toda una tradición y costumbre detrás que indica cómo comportarse, sin necesidad de grandes interpretaciones.

L. Mantenimiento de pautas (latencia): se trata de asegurar la adhesión a largo plazo según ciertos valores compartidos que sirven de marco de referencia distintivo para la acción: las pautas de comportamiento comunes. La socialización y la aculturación (en sentido amplio) son los principales procesos, buscando garantizar una base de identidad para el sistema. Se encarga de ello el sistema cultural. La acción aquí requiere de poca interpretación (baja complejidad simbólica), pero alta contingencia, porque compartir ciertos valores no restringe el campo de cursos de acción a seguir tanto como para hacerlos previsibles -de ahí la importancia que se plasmen en un orden normativo específico-.

\section{ILUSTRACIÓN 1}

Esquema AGIL

\begin{tabular}{|c|c|c|c|}
\hline$\hat{4}$ & & Fines & Medios \\
\hline & Externo (situación) & $\rightarrow \mathrm{G}$ & $\rightarrow \mathrm{A}$ \\
\hline $\begin{array}{l}\text { Complejidad } \\
\text { simbólica }\end{array}$ & & Logro de metas & Adaptación \\
\hline & Interno & $\begin{array}{l}\text { Integración } \\
\rightarrow 1\end{array}$ & $\begin{array}{l}\text { Mantenimiento de } \\
\text { pautas } \\
\rightarrow \mathrm{L}\end{array}$ \\
\hline & Contin & a de la acción & \\
\hline
\end{tabular}

Fuente: Elaboración propia con base en Almaraz (1979) y Münch (1980).

La función de integración implica la creación y sostenimiento de una solidaridad básica entre los actores sociales, en un sentido similar al de Durkheim (Parsons, 1977: 250; 2007: 
56; Sciortino, 2005)4 . A Parsons lo mueve una preocupación por el orden social: ¿cómo reducir la contingencia ligada a las relaciones con el ambiente y a la interpretación (de aquel y de los valores abstractos compartidos), de modo de lograr una convergencia estable de la sociedad? El sistema social tiene que traducir los valores en normas concretas, que especifiquen cursos de acción concretos, y de ese modo organizar las relaciones internas (Münch, 1994; Parsons, 2007).

Sistema social no es necesariamente igual que sociedad, siendo esta un tipo particular de aquel, caracterizado por la autosuficiencia en relación con la organización y los recursos internos como al provecho obtenido de su ambiente para realizar su cultura normativa (De Marinis, 2010b; Parsons, 1974a). En el análisis del sistema social, con el énfasis de las teorías cibernéticas en los sistemas integrados, es posible aplicar nuevamente el esquema AGIL, como se ve en la llustración 2.

\section{ILUSTRACIÓN 2}

Esquema AGIL para el sistema social

\begin{tabular}{|c|c|}
\hline Economía & Política \\
\hline Sistema fiduciario & Comunidad Societal \\
\hline
\end{tabular}

Fuente: Elaboración propia con base en Almaraz (1979) y Münch (1980).

Repitiendo la lógica del modelo previo, reconocemos aquí nuevos subsistemas, ligados a funciones del sistema social (Gerhardt, 2001). A la economía le corresponde el desarrollo y asignación de recursos básicos, cuyo medio de intercambio generalizado es el dinero. La tarea de la política es la coordinación de esfuerzos para metas colectivas, cuyo medio de intercambio generalizado es el poder. La transmisión, mantenimiento y desarrollo de valores compartidos es tarea del sistema fiduciario, cuyo medio de intercambio generalizado son los compromisos de valor. Finalmente, aparece aquí la CS, núcleo integrativo del sistema social, estableciendo marcos compartidos de órdenes normativos (leyes, costumbres, tradiciones, etc.) para delimitar el campo de acción y establecer las bases de confianza y solidaridad entre actores.

4 Sasín (2012: 91-93) observa que esta estrategia duplica el problema de la integración, del sistema social a la CS. 
La integración indica un impulso inherente a la solidaridad, un sentimiento de pertenencia conjunta, impulso regulado por normas para reducir la contingencia de la subjetividad. Esta solidaridad rebasa el respeto mutuo y la contribución al bien colectivo, remitiendo a una identificación mutua (Parsons, 2007: 56). Frente a la fuerza centrífuga de la individualidad, la integración responde como la fuerza centrípeta que, anclada en valores y creencias colectivas, traduce ideas en normas concretas que orientan la acción (Gerhardt, 2002; Parsons, 2007). Su medio de intercambio generalizado es la influencia, que es la capacidad de hacer que otros tomen las decisiones esperadas, buscando su anuencia, sea porque lo asume como algo bueno o porque teme las sanciones de comportarse de otro modo (Gerhardt, 2001). Así, la eficacia de la influencia expresa la confianza en los lazos solidarios detrás de quien ejerce la influencia: nos dejamos influir porque creemos en quien lo hace (Alexander, 1989). Es la función cibernética de control para lograr la autosuficiencia.

Esta expansión de su análisis de los medios generalizados de intercambio a diferentes subsistemas, se basa en las analogías a partir del dinero, con un extenso desarrollo respecto del medio del poder (Parsons, 1977: 204-214). Luhmann utiliza explícitamente esta base para expandir la importancia del poder como medio simbólico de restringir las selecciones de la comunicación, criticando la efectividad que Parsons le atribuye a este medio, pues su carácter relacional es el origen de la posibilidad de su deflación (Torres Navarrete, 2004: 93-103). Habermas (1998: 102; 2001: 373-374), por su parte, encontrará excesivo y erróneo este desarrollo del argumento: aunque el dinero y el poder dan cuenta de la integración en los subsistemas económico y político, su intromisión en la esfera del mundo de la vida no parece legítima ni fundada.

\section{LA CS EN EL MARCO EVOLUCIONISTA}

Basado en la trayectoria previa, Parsons presentó entonces a la CS dentro de su estudio acerca del cambio en las sociedades, aspecto rezagado en su obra ${ }^{5}$. Así, en La sociedad: perspectivas evolutivas y comparadas (1966) retoma el problema hobbesiano del orden, planteado en su primera obra, para introducir su "solución" por medio de la CS (1974a: 18). La define como: el núcleo de una sociedad, como sistema, es el orden normativo, organizado dentro de un patrón, en el que se organiza colectivamente la vida de una población. Como orden, contiene valores y normas diferenciadas y particularizadas, así como reglas, que requieren referencias culturales para resultar significativas y legítimas. Como colectividad, despliega un concepto organizado de membrecía que establece una distinción entre los individuos que pertenecen o no a ella (Parsons, 1974a: 24).

Quedan así presentadas las líneas fundamentales de lo que Parsons entendía por CS: una lealtad básica que mantenga unida la pluralidad (Parsons, 1974b: 23). Siguiendo el rastreo que realiza Alexander (2005), la CS, como núcleo del sistema social, se desdobla sucesivamente en:

5 Fox et al. (2005) y Gerhardt (2001) entienden que llega aquí como efecto de la reflexión respecto de los medios generalizados de intercambio. 
1. Orden: su relevancia fundamental, en continuidad con anteriores planteos, remite a la garantía del orden (estabilidad) de la sociedad. Al modo de la solidaridad durkheimiana, debe establecer los lazos básicos de integración y lealtad entre actores, tanto en su semejanza (mecánica) como en su diferencia (orgánica) (De Marinis, 2010b; Sciortino, 2005). Incluye tanto la "promoción" de ciertas pautas comunes como la corrección de aquellas desviadas.

a. Valores: la referencia constitutiva del orden son los valores compartidos del sistema social, los que guían las pautas básicas de comportamiento.

a.1. Generales: la vida en sociedades complejas (modernas) no puede guiarse por la aplicación de reglas en exceso específicas, a riesgo de resultar excluyentes. Patrones generales de valoración sirven de principios morales como marco universal de justicia (Gerhardt, 2002). Se incluye bajo este contexto la referencia a nociones últimas de la realidad (Parsons, 1974a: 19). Se trata de una cultura compartida.

a.2. Particulares: al mismo tiempo, los valores deben encontrar expresiones específicas entre los distintos grupos sociales, que no contradigan los principios generales. De otro modo, el marco de referencia sería demasiado contingente como para garantizar el orden: se requieren especificaciones particularizadas, según la naturaleza del actor social considerado (su posición y rol, relación con otros actores, etc.). Se trata de aspectos particularizados de la cultura.

b. Reglas: ambos conjuntos de valores se deben expresar en un sistema de normas, formales e informales, que garanticen la menor complejidad simbólica de los mismos. Se trata de incrementar la capacidad del sistema de controlar su organización interna, delimitar el campo de lo asequible y fomentar ciertos comportamientos.

2. Colectividad: no trivialmente, la CS define grados de membrecía, según la organización de un núcleo y la existencia de otros actores (individuales o colectivos) que pertenecen al sistema social sin ser parte plena de la CS. Se definen, a fin de cuentas, grados de ciudadanía ${ }^{6}$. Parsons asocia abiertamente en la CS a la solidaridad en términos durkheimianos con la idea de ciudadanía de T.H. Marshall (Parsons, 1974b: 33; 2007: 151).

En términos generales, para Parsons (1974a: 24-36; 2007: 76-77) la CS se compone de normas que depositan valores (especificación de abstracciones), y en los que se constituye la referencia fundamental para los subsistemas político y económico (control de la contingencia y la complejidad simbólica), responsables por la satisfacción de necesidades sociales: el logro de metas comunes y la asignación de recursos (control de la tecnología). Las relaciones con los demás subsistemas quedan de plano realzadas: la legitimidad de un orden se basa en su justificación en un conjunto de valores (subsistema cultural); su persistencia requiere de lograr las correctas motivaciones de los actores mediante los procesos de socialización

6 Luhmann (1998: 176-181) señalará que estos grados de ciudadanía componen una estratificación social compleja basada en esquemas de exclusión no uniformes: la pertenencia se regula por la participación autónoma en los diferentes subsistemas funcionales. 
(subsistema de la personalidad), tanto como su corrección en caso de desvío, y la garantía del suministro de los requisitos básicos para la vida (Parsons; 1974b: 25-29). Si miramos con atención, estas relaciones son expresiones renovadas (tras 15 años) de los prerrequisitos funcionales del sistema social.

La CS es el núcleo estable de la sociedad y, por tanto, la llave para la puerta al cambio: sin perjuicio de las modificaciones que ocurran, existe un "centro gravitacional" que sostiene al conjunto social. Quizás esta sea una influencia de la disciplina de origen de Parsons, la economía, con su análisis de equilibrios sucesivos; no se trata de negar el cambio, sino de analizar las tendencias a la estabilidad (Parsons, 2007: 147). La dicotomía clásica ha desaparecido: la comunidad ya no expresa un tipo empírico distinguible de la sociedad, sino que expresa su núcleo estructural (sirve como tipo analítico); la comunidad ya no precede en el tiempo a la sociedad, sino que toda sociedad tiene un núcleo comunitario (lo que le permite aplicarla a toda la historia, desde una perspectiva de evolución). La comunidad ya no remite a imágenes de relaciones cercanas, afectivas, cara a cara, es el complejo analítico que funda la permanencia de la sociedad (De Marinis, 2010b).

Desde una óptica evolutiva, las inconsistencias entre valores, normas, creencias e intereses son la fuente del cambio social (Mitchell, 1967). El cambio modernizador implica una creciente diferenciación funcional, donde las unidades o los subsistemas ganan en capacidad de adaptación a (nuevos) problemas más específicos. Procesar ese cambio afecta en distinto grado a los diferentes grupos sociales, alterando la estructura social de conjunto (Parsons, 1967a). Si estos grupos ofrecen resistencia, se puede producir un cambio regresivo. Si la diferenciación logra prevalecer, el sistema como tal es capaz de atender más necesidades, en un sentido de especialización del trabajo. Esto implica unidades más diferenciadas, cuya integración se vuelve desafiante, porque ya no alcanza con una identidad básica sostenida en la semejanza.

Así, el proceso de cambio como diferenciación implica (Alexander, 1989; Parsons, 1974b, 2007): i) instituciones cada vez más especializadas, que no es lo mismo que más autónomas: al contrario, dependen cada vez más unas de otras, y por ello es necesario garantizar su ligazón mutua; ii) un criterio más general de pertenencia a la comunidad, que admita la membrecía a base de competencias mínimas, y asigne derechos y obligaciones no particulares; y iii) valores que se abstraen cada vez más para permitir el consenso. La CS regula este proceso en la medida en que procesa estas modificaciones, como necesidad funcional. La necesidad de superar los "clivajes" de los grupos diferenciados remite a un proceso de pluralismo institucional, junto con una ampliación de la ciudadanía plena (Gerhardt, 2001; Parsons, 1974b). Parsons (2007: 59) busca así las bases consensuales tácitas de ese "nosotros" que lo preocupa desde sus obras tempranas.

\section{TENSIONES INTERNAS A LA CS}

Existen algunas tensiones que Parsons tematizó sin extenderse, a riesgo de encontrar dificultades en sostener su noción de CS como paradigma de la solidaridad (Parsons, 2007: 
148). Resultan particularmente relevantes dos elementos internos: la existencia de grupos sociales no miembros de la CS y el uso de la coerción 7 . Llamamos la atención acerca de ellos porque entendemos que componen el núcleo del problema no resuelto de Parsons, esto es, el problema del orden. Debido a su creciente interés por explicar ese orden utilizando como modelo la sociedad estadounidense de postguerra, la cuestión de los valores democráticos que supuestamente encarna esta propuesta conceptual no es trivial. Veamos.

\section{Grupos no miembros}

En el esquema simplificado de diferenciación, los nuevos grupos son incorporados por una necesidad funcional del sistema, tendiendo a pluralismo de modo... natural (Sciortino, 2005: 116). En primer lugar, esto soslaya el papel activo de los grupos para lograr su inclusión, reavivando la crítica formulada por Therborn (1973) respecto de que en el esquema parsoniano parece que nadie efectivamente hace algo. No obstante, sería injusto decir que no es posible incorporar esta dimensión a su esquema: de hecho, Sciortino (2005: 124-125) insiste en este punto, al realzar que Parsons identifica el potencial conflicto entre grupos incluidos y grupos buscando inclusión. El pluralismo, más allá de una necesidad funcional, es el resultado de la acción organizada. No resulta demasiado arriesgado entender a distintas luchas de minorías como requerimientos por inclusión; sin embargo, resulta problemático no reconocer las distintas dotaciones de recursos, estrategias y capacidades de los diferentes grupos. Esto significa que no todos los grupos obtendrían el mismo nivel de inclusión ni a la misma velocidad, aspectos en los que Parsons no nos ofrece explicaciones.

Esto nos lleva al segundo punto, que es la distinción misma entre el núcleo de miembros originales y los nuevos grupos buscando membrecía. Aun cuando la incorporación requiere de la abstracción y generalización de las pautas de valores y su expresión normativa (Parsons, 1974a: 42), no puede evitarse registrar que existe un núcleo original de valores a abstraer. Aunque no se trataría de la imposición de valores particularistas del grupo núcleo original, resulta ingenuo suponer que se trata de una integración democrática, así como lo hace Parsons (2007: 58-59) al afirmar el ideal consensual que guía la CS, a diferencia de la obligatoriedad política.

Alexander (2005) señala aquí una tensión entre la necesidad de integración y la búsqueda de justicia (criterios universales de acción que eviten la vulneración de otros [Parsons, 1974a: 30]. La "jerarquía moral" de los miembros originales se expresa en la universalización de sus pautas para incluir (potencialmente) a los nuevos miembros. Esto expresa justamente lo que en otras corrientes de pensamiento se conoce como construcción de hegemonía, esto es, la prevalencia de un conjunto particular de valores y creencias respecto del conjunto de la población (Gramsci, 1975; Laclau y Mouffe, 1985). La construcción de consenso requerida por el proceso democrático de legitimación involucra crear un sentido común, una tradición, que guíe la acción en ciertos sentidos.

7 Se pueden consultar otros desarrollos de tensiones y contradicciones en Sasín (2012). 
La legitimación, en el marco parsoniano, implica que el grupo "entrante" tiene que lograr las mismas condiciones de humanidad que los miembros originales, en algún sentido o categoría que puede ser nuevo (Gerhardt, 2001: 194). Por eso es que Parsons asoció directamente el proceso de expansión de la ciudadanía plena con la tipología de derechos de Marshall (Cohen y Arato, 2000; Parsons, 1967b, 2007; Münch, 1994). Esto es en parte el resultado de la idealización de Parsons de la etapa de la historia que vive el welfarismo estadounidense (ver, por ejemplo, Parsons, 2007: 74-75). Sin embargo, la ampliación de la ciudadanía plena a nuevos grupos puede "chocar" con los intereses, valores o creencias del núcleo de membrecía original.

De hecho, en los años que Parsons está desarrollando estas ideas, la organización keynesiana del sistema mundo capitalista comienza a resquebrajarse, entrando en una fase de crisis que anulará o retraerá la mayor parte de los derechos ganados durante esta etapa (Dumenil y Levi, 2007). Sasín (2012: 78) afirma que la aparición misma del concepto de CS "(...) podría parecer estar más motivada en los condicionantes del contexto epocal que en desarrollos intrínsecos o necesidades inherentes a la construcción de la teoría [de Parsons]". A fines de los sesenta, la ampliación de la ciudadanía chocó en términos materiales (e ideológicos) con las necesidades de reproducción del capital, e indujo a una nueva etapa definida genéricamente como neoliberalismo (Astarita, 2006). El nuevo "consenso" moral se construyó sobre la base de ciertos valores identificables con ciertas clases, sus necesidades e intereses. Por tanto, no resulta trivial la definición de quienes componen el núcleo de membrecía original.

\section{Uso de la coerción}

La segunda tensión es el rol de la coerción. Aun cuando Parsons buscó desde el comienzo dar cuenta de una salida para explicar el orden sin necesidad del recurso coercitivo, se encuentra aquí con un traspié. Justo después de explicar el criterio de justicia, Parsons (1974a: 30) dice que "(...) la prevención última de la acción destructiva [sobre la CS] es mediante el empleo de la fuerza física (...). El control o la neutralización del uso organizado de la fuerza es una necesidad funcional para el mantenimiento de una comunidad societaria". Es decir, la garantía última del orden, la estabilidad, la integración, es el uso de la fuerza (1974b: 26-27), aunque esta sea un medio residual para disuadir, del que no se puede abusar sin hacerle perder eficacia (1967c: 272).

El uso de la fuerza es controlado por el subsistema político (Parsons, 1974a: 33). Según se nos dijo, este está ordenado en referencia a un conjunto de normas que expresan valores, que -según discutimos en el inciso anterior- responden primaria, aunque no exclusivamente, a un grupo o conjunto de grupos sociales. Es decir, la fuerza garantiza en última instancia un orden fundado en los valores de algunos. Claro que esa garantía solo aplica como último recurso, pues su uso sistemático produciría una devaluación de su capacidad de sostener el orden (1967c: 296). Parsons (1974a: 42) señaló, frente al marxismo, que suponer el monopolio de un grupo en la posición de membrecía real es una dicotomía excesivamente simplificada. Pero no pudo ofrecer razones teóricas por las que esta posición monopólica 
o cuasimonopólica no podría sostenerse: ¿qué evita que el grupo constituyente original no aproveche su posición privilegiada para contener, administrar o dosificar el acceso de otros grupos? ¿Qué evita que utilice el recurso coercitivo a este fin, aunque solo lo haga en situaciones límite? ${ }^{8}$

Quizás por esto, a lo largo de su obra, Parsons reconoció las limitaciones de la orientación normativa y la necesidad de garantizar ciertos medios de control social (Boaso, 1980; Mitchell, 1967). Esto lo llevó a explicitar, desde el principio hasta el final de su carrera, la importancia de la educación como medio de socialización para inculcar ciertos valores particulares (Parsons, 1967a: 206-238; 1986: 57). Cuando en El sistema social explicitó los prerrequisitos funcionales, como ya discutimos, también realzó la importancia de la correcta motivación, y la "corrección de conductas lesivas". Pero inclusive más atrás en el tiempo, y en relación con el problema hobbesiano como lo formulamos al principio de este trabajo, ya Alexander (2005) detectó que la salida propuesta por Parsons en la Estructura de la acción social es no democrática. El problema con la imposición coercitiva externa es su baja eficacia: de allí la importancia de la internalización de un cierto orden normativo ${ }^{9}$. La falta de reconocimiento de Parsons acerca de esta tensión no es una ingenuidad. En caso de afirmarla, caería su ilusión democrática y pluralista de la sociedad (estadounidense) moderna ${ }^{10}$.

\section{COMENTARIOS FINALES}

Talcott Parsons ofreció una intuición innovadora en relación con la problemática de la comunidad, permitiendo un pasaje a la comprensión de su actualidad en sociedades complejas. El giro que implicó abandonar su polaridad con la sociedad anunció su incorporación sistémica al funcionamiento y estabilidad de las sociedades. Ofreció así una explicación al orden social, pero también -y esto no es menor- un programa de acción: cómo reforzar un orden social dado. Según el autor, la acción autointeresada del individuo se limita por las normas compartidas y su efectividad. Según Münch (1983), esta depende del grado de inclusión en la comunidad, los lazos afectivos, la claridad y conciencia de las normas y el refuerzo por medio de rituales y ceremonias. Estas serían pues las áreas en las que intervenir para desarrollar el orden y contener tensiones en sociedades crecientemente complejas.

La omisión teórica de Parsons puede ubicarse en la negación a los aspectos no plenamente democráticos de su propuesta, o mejor dicho, a los límites de su propuesta de democracia. Se trata de la distinción entre grupos miembros y no miembros, y el problema del uso de la coerción. Debemos enfatizar que en ambos casos se trata de una crítica interna: lo que

8 A esta cuestión refiere la noción marxista de dictadura de clase, y no al régimen político -que puede ser democrático (Balibar, 1977)-.

9 Por ello, Dawe (1970) afirma que el planteo de Parsons sigue planteando una restricción externa al actor, cuya internalización no hace sino afirmar la exterioridad.

10 La pluralidad es constituida sobre la base de la exclusión de aquellos elementos que cuestionan los fundamentos normativos de la sociedad. Parece que, aun conociendo y estudiando estos fenómenos, no incorpora orgánicamente a la constitución del "pluralismo" americano la década antirroja de los 20 (con hitos como el asesinato de Sacco y Vanzetti) o el macartismo (Parsons, 2007). Sin perjuicio de la "inteligencia" funcional del sistema, esto es un cuestionamiento a sus bases democráticas. 
Parsons trató de defender una y otra vez en su obra, termina sin solución. La propuesta de CS puede ser útil para pensar no solo los años de la expansión capitalista de posguerra, sino la propia actualidad. Pero, claro, para ello es necesario reconocer los aspectos no democráticos, de asimetría de poder político, contenidos de la misma.

\section{BIBLIOGRAFÍA}

Alexander, J. (1989): Las teorías sociológicas desde la Segunda Guerra Mundial, Gedisa, Madrid.

(1990): La centralidad de los clásicos, en A. Giddens, J. Turner y otros, Teoría social hoy, Alianza, Madrid.

(2005): Contradictions in the Societal Community: The promise and disappointment of Parsons' concept, en R.C. Fox, V. Lidz y H.J. Bershady (eds.): After Parsons: A theory of social action for the twenty first century, Russell Sage Foundation, Nueva York, pp. 93-110.

Almaraz, J. (1979): La transición del modelo interactivo al sistémico en Parsons, Reis, 8, pp. 5-32.

Astarita, R. (2006): Valor, mercado mundial y globalización, Kaicrón, Buenos Aires.

Boasso, C. (1980): El estructuralismo funcional de Parsons, Instituto de Ciencias Sociales, Buenos Aires.

Balibar, E. (1977): Sobre la dictadura del proletariado, Siglo XXI, México D.F.

Cohen, J. y A. Arato (2000): Sociedad Civil y teoría política, Fondo de Cultura Económica, México D.F.

Dawe, A. (1970): The two sociologies, British Journal of Sociology, 2 (21), pp. 207-218.

De Marinis, P. (2005): 16 comentarios sobre la(s) sociología(s) y la(s) comunidad(es), Papeles del Centro de Estudios sobre la Identidad Colectiva (CEIC), 15.

(2010a): Estado y comunidad, de los clásicos a Parsons (y más allá), Propuesta Educativa, 19 (33).

(2010b): La comunidad societal en Talcott Parsons: un ejercicio para poner a prueba ciertas hipótesis de lectura acerca de la historia del concepto de comunidad en la teoría sociológica, presentado en las VI Jornadas de Sociología de la UNLP, La Plata, 9 y 10 de diciembre.

Duménil, G. y Dominique L. (2007): Crisis y salida de la crisis. Orden y desorden neoliberales, Fondo de Cultura Económica, México D.F.

Fox, R.C., V. Lidz y H.J. Bershady (eds.) (2005): After Parsons: A theory of social action for the twenty first century, Russell Sage Foundation, Nueva York.

Gerhardt, U. (2001): Parsons' analysis of the societal community, en J. Treviño, (ed.): Talcott Parsons today. His theory and legacy in contemporary sociology, Rowman \& Littlefield Publishers, Lanham.

Gerhardt, U. (2002): Talcott Parsons. An intellectual biography, Cambridge University Press, Cambridge.

Gramsci, A. (1975): Notas sobre Maquiavelo, sobre la política y sobre el Estado moderno, Juan Pablos Editor, México D.F.

Habermas, J. (1998): Facticidad y validez, Trotta, Madrid.

(2001): Teoría de la acción comunicativa, vol. 2, Taurus, Madrid.

Holton, R. y B. Turner (1986): Talcott Parsons on economy and society, Routledge, Londres y NuevaYork.

Laclau, E. y C. Mouffe (1987): Hegemonía y estrategia socialista. Hacia una radicalización de la democracia, Siglo XXI, Madrid. 
Luhmann, N. (1995): Introducción a la teoría de sistemas, Universidad Iberoamericana, México D.F. (1998): Complejidad y modernidad: de la unidad a la diferencia, Trotta, Madrid.

Mitchell, W. (1967): Sociological analysis and politics. The theories of Talcott Parsons, Prentice-Hall, Nueva Jersey.

Münch, R. (1982): Talcott Parsons y la teoría de la acción. La constitución del núcleo kantiano, Revista Internacional de Sociología, 41, pp. 51-85.

(1983): From pure methodological individualism to poor sociological utilitarism: a critique o fan avoidable alliance, The Canadian Journal of Sociology, 8 (1), pp. 45-77.

(1990): Teoría parsoniana actual: en busca de una nueva síntesis, en A. Giddens, J. Turner y otros: Teoría social hoy, Alianza, Madrid.

(1994): La interpenetración de la microinteracción y las macroestructuras en un orden institucional complejo y contingente, en J. Alexander, B. Giesen, R. Münch y N. Smelser (comps.): El vínculo Micro-Macro, Gamma, Guadalajara.

Nisbet, R. (1996): La formación del pensamiento sociológico 1, Amorrortu, Buenos Aires.

Parsons, T. (1966): El sistema social, Revista de Occidente, Madrid.

(1967a): Ensayos de teoría sociológica, Paidós, Buenos Aires.

(1967b): Full citizenship for the Negro American?, en T. Parsons: Sociological theory and modern society, The Free Press, Nueva York, pp. 422-465.

(1967c): Some reflections on the place of force in social process, en T. Parsons: Sociological theory and modern society, The Free Press, Nueva York, pp. 264-296.

(1968): Estructura de la acción social, Guadarrama, Madrid.

(1974a): La sociedad: perspectivas evolutivas y comparadas, Trillas, México D.F.

(1974b): El sistema de las sociedades modernas, Trillas, México D.F.

(1977): Social systems and the evolution of action theory, The Free Press, Nueva York.

(1986): Biografía intelectual. El simbolismo económico y religioso en Occidente, Universidad Autónoma de Puebla, Puebla.

(2007): American Society. A theory of the societal community, Paradigm, Londres.

Sasín, M. (2012): ¿Por qué le habrá puesto "comunidad"? Una aproximación al lugar de la comunidad societal en la teoría de Talcott Parsons, Sociológica, 27 (77), pp. 71-108.

Sciortino, G. (2005): How different can we be? Talcott Parsons, the societal community and the multicultural debate, en R.C. Fox, V. Lidz y H.J. Bershady (eds.): After Parsons: A theory of social action for the twenty first century, Russell Sage Foundation, Nueva York, pp. 111-136.

Sciortino, G. (2007): Introduction: The action of social structure, en T. Parsons: American Society. A theory of the societal community, Paradigm, Londres, p. 1-21.

Therborn, G. (1973): Social practice, social action, social magic, Acta Sociológica, 16 (3), pp. 157-174.

Torres Navarrete, J. (2004): Luhmann: La política como sistema, Fondo de Cultura Económica, México D.F. 